\title{
Infective endocarditis in patients with pyogenic spondylodiscitis: implications for diagnosis and therapy
}

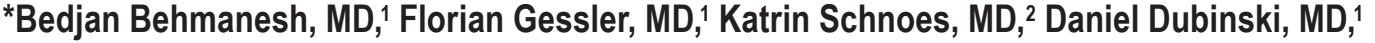 \\ Sae-Yeon Won, MD, ${ }^{1}$ Jürgen Konczalla, MD, PhD, ${ }^{1}$ Volker Seifert, MD, PhD, ${ }^{1}$ Lutz Weise, MD, PhD, ${ }^{1,3}$ \\ and Matthias Setzer, MD, PhD ${ }^{1}$
}

\begin{abstract}
1'Department of Neurosurgery, and 2Medical Clinic III, Department of Cardiology, Goethe University Hospital, Frankfurt am Main, Germany; and ${ }^{3}$ Division of Neurosurgery, Dalhousie University Halifax, QEIl Health Sciences Centre, Halifax, Nova Scotia, Canada
\end{abstract}

\begin{abstract}
OBJECTIVE The incidence of patients with pyogenic spinal infection is increasing. In addition to treatment of the spinal infection, early diagnosis of and therapy for coexisting infections, especially infective endocarditis (IE), is an important issue. The aim of this study was to evaluate the proportion of coexisting IE and the value of routine transesophageal echocardiography (TEE) in the management of these patients.

METHODS The medical history, laboratory data, radiographic findings, treatment modalities, and results of TEE of patients admitted between 2007 and 2017 were analyzed.

RESULTS During the abovementioned period, 110 of 255 total patients underwent TEE for detection of IE. The detection rate of IE between those patients undergoing and not undergoing TEE was 33\% and 3\%, respectively $(p<0.0001)$. Thirty-six percent of patients with IE needed cardiac surgical intervention because of severe valve destruction. Chronic renal failure, heart failure, septic condition at admission, and preexisting heart condition were significantly associated with coexisting IE. The mortality rate in patients with IE was significantly higher than in patients without IE $(22 \%$ vs $3 \%$, $p=0.002)$.
\end{abstract}

CONCLUSIONS TEE should be performed routinely in all patients with spondylodiscitis.

https://thejns.org/doi/abs/10.3171/2018.10.FOCUS18445

KEYWORDS infective endocarditis; spinal infection; surgery; transesophageal echocardiography

$\mathrm{T}$ HE incidence of spondylodiscitis in Western countries has been rising over the past several decades. The main reasons for this are demographic changes, extended life expectancy, and improved access to medical services. ${ }^{2,5,11,19}$ Moreover, the median age of patients suffering from spondylodiscitis is increasing and it is not surprising that a high number of these patients are harboring a significant number of comorbidities. Infective endocarditis (IE) and coexisting pyogenic spondylodiscitis, mostly treated in internal medicine departments, is described in a few case series..$^{12,14,16}$ However, the incidence of and risk factors for IE and outcomes in patients admitted with pyogenic spondylodiscitis are still unclear. Depending on the focus of the primary infection, many patients initially present to surgical departments for treatment of the spinal infection focus. ${ }^{9}, 11$ IE is a life-threatening disease and is associated with high mortality rates of up to $30 \%$. Therefore, early diagnosis and appropriate therapy is crucial, not only of the spinal infection but also of IE. , $, 710,13,21^{\text {Referral of pa- }}$ tients with IE to intensive care units and/or cardiac surgery departments is a life-saving measure, as has already been presented in prospective studies. ${ }^{10,13}$

The aim of the present study was to highlight the incidence of IE and the risk factors and clinical outcomes in patients with pyogenic spondylodiscitis initially admitted to a university spine surgery unit. We further evaluate the value of routinely performed transesophageal echocardiography (TEE) in the management of patients for the

ABBREVIATIONS $\mathrm{Cl}=$ confidence interval; $\mathrm{CRP}=\mathrm{C}$-reactive protein; IDU = intravenous drug use; $\mathrm{IE}$ = infective endocarditis; $\mathrm{OR}=$ odds ratio; $\mathrm{TEE}=$ transesophageal echocardiography; $\mathrm{WBC}=$ white blood cell.

ACCOMPANYING EDITORIAL DOI: 10.3171/2018.10.FOCUS18587.

SUBMITTED August 23, 2018. ACCEPTED October 22, 2018.

INCLUDE WHEN CITING DOI: 10.3171/2018.10.FOCUS18445.

${ }^{*}$ L.W. and M.S. contributed equally to this work. 


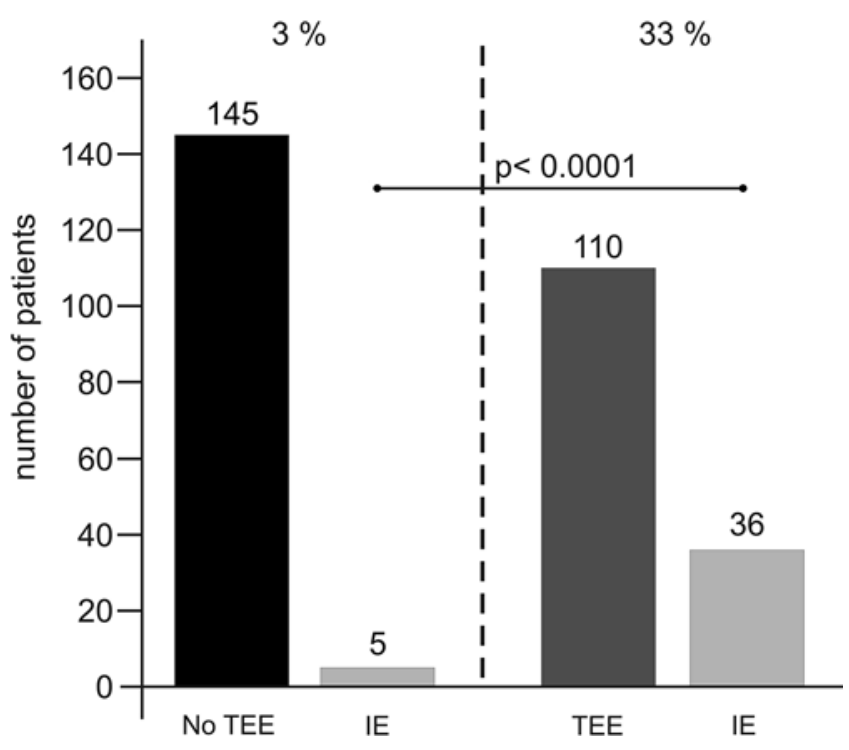

FIG. 1. Bar graph showing the incidence of IE among patients undergoing TEE before and after 2012.

detection of IE. To the best of our knowledge, the routine use of TEE in patients with spondylodiscitis has not been previously reported.

\section{Methods}

All patients admitted to the Department of Neurosurgery in Frankfurt with newly developed pyogenic spondylodiscitis were prospectively entered into an institutional database. An analysis was performed for all cases treated for spinal infection between 2007 and 2017.

TEE performed by a senior cardiologist was implemented as a routine diagnostic tool after 2012, so there was a period before implementation of TEE and a period after implementation. In cases of evident IE, patients were introduced to the department of cardiac surgery for evaluation of the need for cardiac surgical procedures. In cases of conservative therapy for IE, patients were monitored by a cardiologist to detect and evaluate the success of antiinfective therapy and cardiac function. This study was approved by the local ethics committee of the authors' institution. Patient consent was obtained.

\section{Statistical Analysis}

Comparison of important baseline characteristics and surgical parameters between the study groups was made using Fisher's exact test for categorical variables and Student t-tests for continuous variables; $\mathrm{p}$ values $<0.05$ (2-tailed) were deemed significant. All analyses were performed using GraphPad statistical software (version 7.0).

\section{Results}

During the study period, 255 patients with newly diagnosed pyogenic spondylodiscitis were admitted at our department. From 2007 to 2012, 145 patients (57\%) were treated for their spinal infection and IE was detected in 5 patients (3\%). After 2012, 110 patients (43\%) were ad-
TABLE 1. Baseline characteristics of patients with spondylodiscitis with and without IE

\begin{tabular}{lccl}
\hline \multicolumn{1}{c}{ Parameter } & No IE $(\%)$ & $\mathrm{IE}(\%)$ & p Value \\
\hline No. of patients & $74(67.3)$ & $36(32.7)$ & \\
\hline Mean age \pm SD, yrs & $68.5 \pm 10.9$ & $70.3 \pm 7.4$ & 0.3 \\
\hline Males & $51(69)$ & $27(75)$ & 0.9 \\
\hline Vertebral level involved & & & \\
\hline Cervical & $16(21.6)$ & $10(27.8)$ & 0.3 \\
\hline Thoracic & $16(21.6)$ & $5(13.9)$ & 0.6 \\
\hline Lumbar & $42(56.8)$ & $21(58.3)$ & 0.7 \\
\hline 1 level & $51(68.9)$ & $24(66.7)$ & 0.6 \\
\hline Multifocal levels & $23(31.1)$ & $12(33.3)$ & 0.6 \\
\hline Diabetes & $26(35.1)$ & $8(22.2)$ & 0.2 \\
\hline Chronic renal failure & $14(18.9)$ & $14(38.9)$ & 0.04 \\
\hline Predisposing heart condition & $7(9.5)$ & $9(25)$ & 0.04 \\
\hline IDU & $1(1.4)$ & $2(5.6)$ & 0.2 \\
\hline Alcohol abuse & $2(2.7)$ & 0 & 0.6 \\
\hline Heart failure & $9(12.2)$ & $11(30.6)$ & 0.03 \\
\hline Spinal epidural abscess & $22(29.7)$ & $14(38.9)$ & 0.4 \\
\hline Dialysis & 0 & $3(8.3)$ & 0.03 \\
\hline Deaths & $2(2.7)$ & $8(22.2)$ & 0.002 \\
\hline Obesity & $9(12.2)$ & $1(2.8)$ & 0.2 \\
\hline Surgery & $52(70.3)$ & $28(77.8)$ & 0.6 \\
\hline Sepsis & $7(9.4)$ & $10(27.8)$ & 0.02 \\
\hline Boldace type indicates stats & & &
\end{tabular}

Boldface type indicates statistical significance.

mitted with the diagnosis of spondylodiscitis and all underwent TEE. Among 110 patients, 36 (32.7\%) revealed signs of IE in TEE (odds ratio [OR] 17.1, 95\% confidence interval [CI] 5.9-50.0, p < 0.0001; Fig. 1).

Of the 36 patients diagnosed with IE, 27 were males $(75 \%)$ and the mean age was $70.3 \pm 7.4$ years. The spinal infection in these patients was located in the cervical spine in 10 patients $(27.8 \%)$, in the thoracic spine in 5 patients (13.9\%), and in the lumbar spine in 21 patients $(58.3 \%)$. Of the 36 patients with diagnosed IE, in 24 (66.7\%) a singlelevel effect of the spine infection was obvious. Baseline characteristics and predisposing conditions are shown in Table 1. The mean age, sex distribution, location of the infection, number of affected levels, prevalence of diabetes, intravenous drug use (IDU), and alcohol abuse were almost similar between patients with and without IE.

Preexisting comorbidities such as heart failure (OR 3.2, 95\% CI 1.2-8.6, $\mathrm{p}=0.03$ ), septic condition at admission (OR 3.7, 95\% CI 1.7-10.7, $\mathrm{p}=0.02$ ), predisposing heart condition (OR 3.2, 95\% CI 1.1-9.4, $\mathrm{p}=0.04)$, chronic renal failure (OR 2.7, 95\% CI 1.1-6.6, $\mathrm{p}=0.04)$, and terminal renal failure with need for dialysis were observed significantly more often in patients with IE. Of the patients with IE, 9 (25\%) had degenerative cardiac valve disease. Of the patients without IE, $6(8.1 \%)$ had native valve disease (degenerative) and 1 patient (1.4\%) had a prosthetic aortic valve. Furthermore, no significant differences were observed regarding diabetes, surgical or conservative treatment of the spinal infection, and obesity (Table 1). TEE- 
TABLE 2. Microbiological and laboratory data

\begin{tabular}{lccc}
\hline \multicolumn{1}{c}{ Variable } & No IE $(\%)$ & IE $(\%)$ & p Value \\
\hline Pathogen identification & $46 / 67(68.7)$ & $24 / 29(82.7)$ & 0.2 \\
\hline Gram-positive & $39(84.8)$ & $18(75)$ & 0.3 \\
\hline Gram-negative & $7(15.2)$ & $6(25)$ & 0.3 \\
\hline CRP & $12.9 \pm 12.1$ & $13.8 \pm 11.6$ & 0.4 \\
\hline WBC & $10.8 \pm 4.7$ & $11.5 \pm 6.2$ & 0.6 \\
\hline Blood culture positive & $23(34.3)$ & $20(69)$ & $\mathbf{0 . 0 0 2}$ \\
\hline
\end{tabular}

Boldface type indicates statistical significance.

related complications, such as injuries of the gastrointestinal tract or bleeding, did not appear in our investigated patient population.

Comparison between the vertebral spondylodiscitis groups with and without IE revealed that the presence of a spinal epidural abscess was more commonly observed in patients with IE. The rate of disease-related 30-day mortality was significantly higher in patients with IE $(22.2 \%$ vs $2.7 \%, \mathrm{p}=0.002$ ).

\section{Microbiology and Laboratory Data}

Detailed microbiological data are shown in Table 2. Microbiological detection of the infective-causing pathogens was achieved in $73 \%$ of all cases. No significant difference was observed in the rate of pathogen identification between groups (OR 0.5, 95\% CI 0.2-1.4, $\mathrm{p}=0.2$ ). Gram-positive pathogens considered to be causative for spondylodiscitis were isolated in $82 \%$ of all cases, more specifically in 39 of 46 patients $(84.8 \%)$ without IE and in 18 of 24 patients (75\%) with IE (OR 0.5, 95\% CI 0.2-1.8, $\mathrm{p}=0.3$ ). Staphylococcus aureus was the most commonly isolated microorganism $(56.3 \%$ vs $37.5 \%$, OR $0.5,95 \%$ CI $0.2-1.4, \mathrm{p}=0.2$ ).

Blood culture tests were positive in 43 patients (44.8\%), including all patients with IE $(20 / 29,69 \%)$ and without IE $(23 / 67,34.3 \%)$ (OR 4.3, 95\% CI 1.7-10.8, $\mathrm{p}=0.002)$. White blood cells (WBCs) and C-reactive protein (CRP) values at admission did not reveal a significant difference between the two groups.

\section{Clinical Relevance and Therapeutic Implication}

Thirteen (36\%) of 36 patients revealed severe valve destruction caused by IE and a subsequent cardiac surgery was recommended in these patients (Fig. 2). Twenty-three patients $(64 \%)$ were treated conservatively. In patients with a recommendation for cardiac surgery the mitral valve was affected in 9 cases and the aortic valve in 4 cases (Fig. 3). All patients revealed insufficient cardiac function due to severe valve destruction. In 9 patients (69.2\%) the recommended cardiac surgical procedure could be performed; $4(30.8 \%)$ of these 13 patients died due to progressive cardiac failure before undergoing cardiac surgery.

\section{Diagnostic Value}

In this study 36 patients with coexisting IE were identified from 110 patients undergoing TEE. Three TEEs were necessary to identify 1 patient with IE, thus harboring a

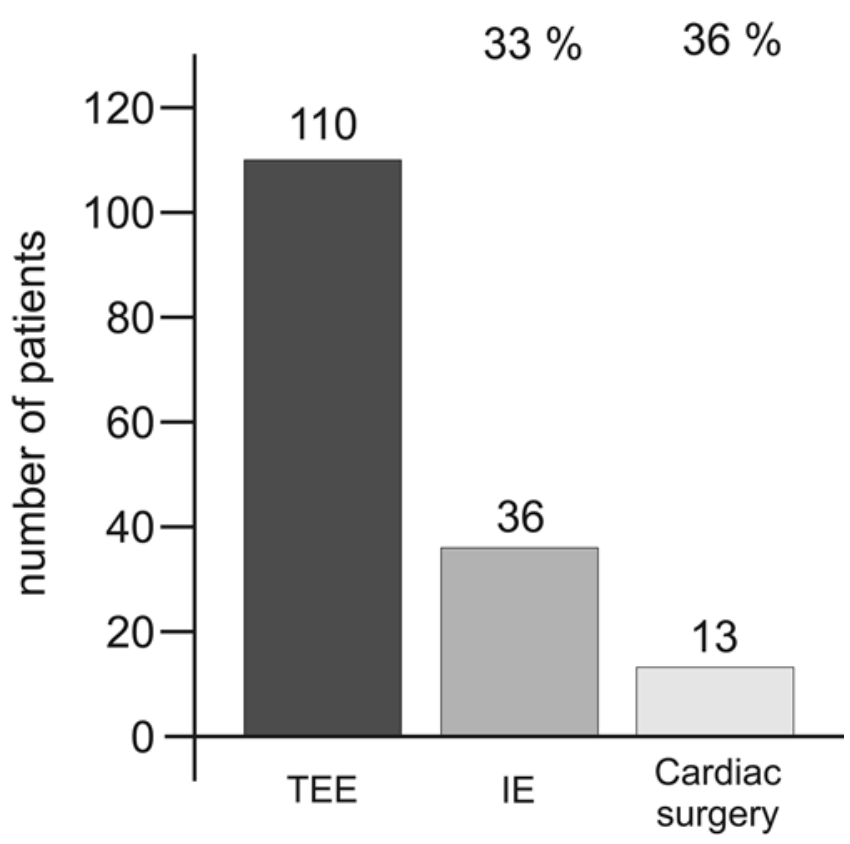

FIG. 2. Bar graph demonstrating the clinical relevance of TEE implementation.

potential risk for valve destruction and cardiac failure. Moreover, by routinely using TEE, 8 TEEs were necessary to identify 1 patient with further need for cardiac surgical intervention (Fig. 2).

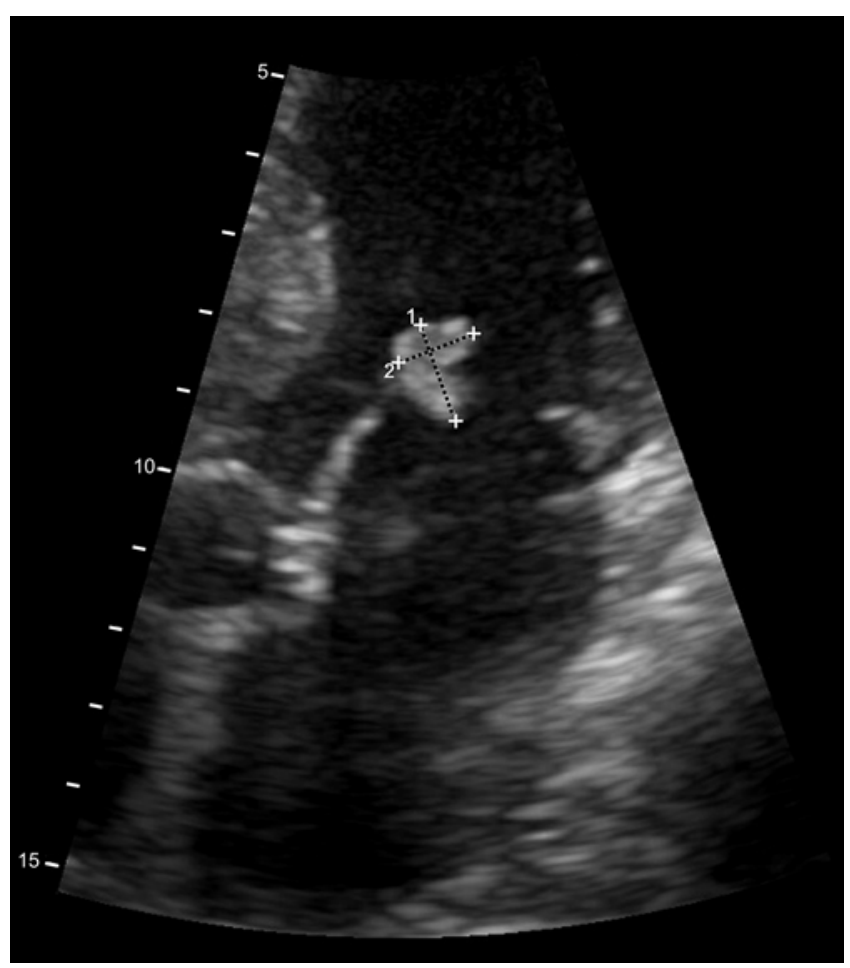

FIG. 3. TEE (ultrasound) demonstrates vegetation (crosshairs) on the valve as a sign of IE. 


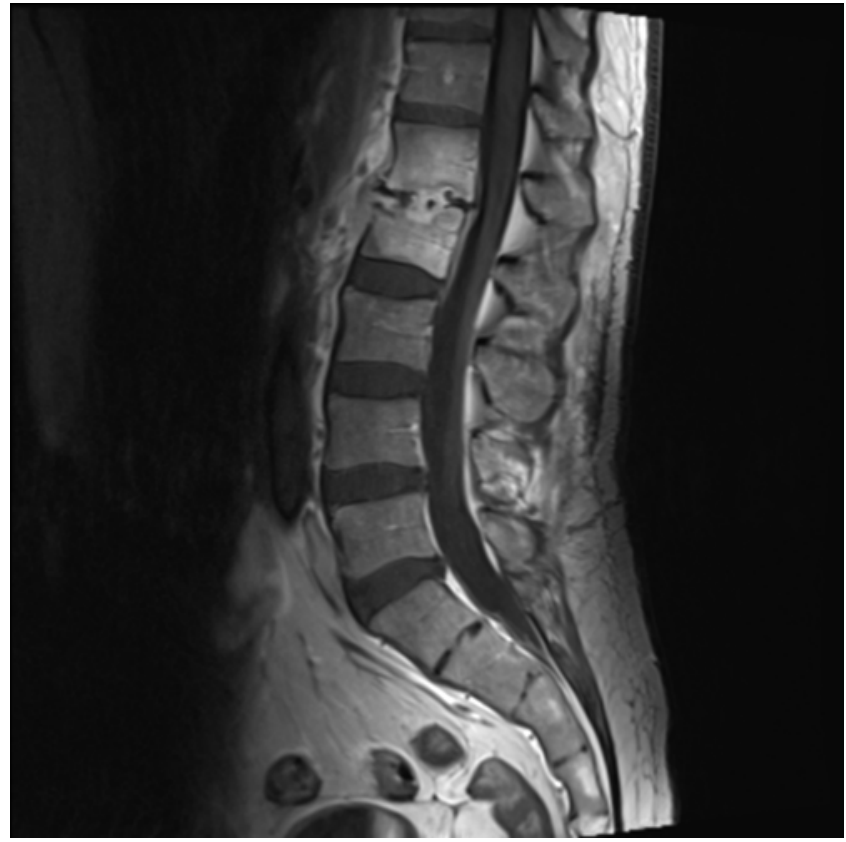

FIG. 4. Sagittal MRI demonstrating spondylodiscitis in a patient harboring coexisting IE.

\section{Discussion}

The early diagnosis of IE is crucial for the initiation of the appropriate antibiotic therapy and to identify patients who may benefit from extended cardiac function monitoring and early surgical intervention. Pyogenic spondylodiscitis remains a severe disease associated with high morbidity (Fig. 4). Within this study, we addressed the value of TEE in identifying IE in patients with pyogenic spondylodiscitis. To the best of our knowledge the routine use of TEE in the diagnostic workup of patients with spondylodiscitis has thus far not been described.

Coexisting pyogenic spondylodiscitis among patients with IE has already been reported from cardiologists. ${ }^{15,16,18,20}$ But routine TEE workup for the detection of IE among patients with spondylodiscitis who present to a surgical department has not been described until now. Because the incidence of pyogenic spondylodiscitis is rising, many spine surgeons undertake measures to improve the clinical outcome of patients with pyogenic spondylodiscitis. Focusing on the treatment of the spinal infection while neglecting coexisting IE may result in poor patient outcomes despite enormous efforts.

The implication of the routine uses of TEE in our investigated patient population resulted in a detection rate of IE of almost 33\%, which means that 3 TEEs were needed to detect 1 patient with IE. To avoid any selection bias, all patients admitted with the diagnosis of spondylodiscitis underwent TEE.

Whether the pyogenic spinal infection was the reason for IE or vice versa was not resolved within this analysis. Next to TEE, blood cultures may be considered indicative for bacteremia associated with IE. ${ }^{4,8}$ In our patient cohort, $67 \%$ of patients with the diagnosis of IE revealed a positive blood culture, which would leave 33\% undiagnosed.
It might be tempting to speculate that without the use of TEE, these patients might not have received treatment for IE and ultimately shown poor outcome.

Specific risk factors for coexisting IE such as cardiac comorbidities, renal failure, dialysis, and septic condition at admission were identified in our investigated patient population. If not routinely performed in patients with newly developed pyogenic spondylodiscitis, TEE may at least be performed in all patients with the abovementioned risk factors as a consequence to the data presented within this study. Analyzing further risk factors such as diabetes, IDU, and obesity, we found no statistically significant difference in patients with and without IE. In contrast, diabetes and obesity were more often found in patients not harboring IE. But further research in a larger patient population could evaluate the effect of diabetes, immune compromise, and immunomodulatory drugs on the incidence of IE and spinal infection.

The microbiological analysis revealed a high incidence of IE in patients with pyogenic spondylodiscitis caused by Staphylococcus or Streptococcus species. These findings are consistent with those in the literature, i.e., both pathogens are described to be among the most common pathogens associated with pyogenic spondylodiscitis. Moreover, both pathogens are commonly observed in patients with IE. $4,8,12$

TEE is invasive, costly, and requires technical and medical resources. ${ }^{1,3}$ But we emphasize the effectiveness of TEE and recommend including the routine use of TEE in the standard diagnostic workup in patients with spinal infection. In contrast to transthoracic echocardiography with a sensitivity of $32 \%$ and specificity of $100 \%$ for detecting IE, the addition of TEE increases the sensitivity to $100 \%$, whereas the specificity remains almost unchanged. ${ }^{3}$ In 36\% of the patients diagnosed with IE, subsequent cardiac surgical intervention was necessary in the course of the disease. Recent studies suggest that early surgical intervention in IE is associated with decreased adverse events, including embolic events and mortality, thus further highlighting the need for diagnosing/ruling out IE in patients with IE. ${ }^{710}$ In many cases cardiac surgery was recommended prior to spine surgery. In these patients, a severe valve destruction with cardiac failure was detected and referral to the intensive care unit was recommended before performing cardiac surgery to avoid any complications resulting from severe cardiac failure. If IE would not have been detected in these patients and spine surgery in the prone position for several hours was performed before stabilizing the cardiac situation, it remains speculative how successful the result of the surgery would be in this patient population. Therefore, we postulate that an appropriate and early assessment of the cardiac function in patients with spondylodiscitis is of interest not only for cardiologists and cardiac surgeons, but also for anesthesiologists and spine surgeons to ensure a good outcome. These results are of special interest because IE in the general patient population displays a high mortality rate. ${ }^{17}$

\section{Conclusions}

IE can be commonly diagnosed in patients with pyo- 
genic spondylodiscitis. The routine implementation of TEE for the detection of IE in these patients was very effective and should be recommended, not only when suspected, but rather as part of routine assessment of comorbidities in patients with newly diagnosed spondylodiscitis. As a consequence, patients with otherwise undetected IE may receive adequate treatment, ultimately resulting in better outcomes.

\section{References}

1. Bai AD, Steinberg M, Showler A, Burry L, Bhatia RS, Tomlinson GA, et al: Diagnostic accuracy of transthoracic echocardiography for infective endocarditis findings using transesophageal echocardiography as the reference standard: a meta-analysis. J Am Soc Echocardiogr 30:639-646, 646. e1-646.e8, 2017

2. Calderone RR, Larsen JM: Overview and classification of spinal infections. Orthop Clin North Am 27:1-8, 1996

3. Fowler VG Jr, Li J, Corey GR, Boley J, Marr KA, Gopal AK, et al: Role of echocardiography in evaluation of patients with Staphylococcus aureus bacteremia: experience in 103 patients. J Am Coll Cardiol 30:1072-1078, 1997

4. Fowler VG Jr, Miro JM, Hoen B, Cabell CH, Abrutyn E, Rubinstein E, et al: Staphylococcus aureus endocarditis: a consequence of medical progress. JAMA 293:3012-3021, 2005

5. Gouliouris T, Aliyu SH, Brown NM: Spondylodiscitis: update on diagnosis and management. J Antimicrob Chemother 65 (Suppl 3):iii11-iii24, 2010

6. Habib G, Lancellotti P, Antunes MJ, Bongiorni MG, Casalta JP, Del Zotti F, et al: 2015 ESC Guidelines for the management of infective endocarditis: The Task Force for the Management of Infective Endocarditis of the European Society of Cardiology (ESC). Endorsed by: European Association for Cardio-Thoracic Surgery (EACTS), the European Association of Nuclear Medicine (EANM). Eur Heart J 36:30753128, 2015

7. Haider K, Pinsky MR: Early surgery for native valve infective endocarditis. Crit Care 17:304, 2013

8. Holland TL, Arnold C, Fowler VG Jr: Clinical management of Staphylococcus aureus bacteremia: a review. JAMA 312:1330-1341, 2014

9. Jensen AG, Espersen F, Skinhøj P, Rosdahl VT, FrimodtMøller N: Increasing frequency of vertebral osteomyelitis following Staphylococcus aureus bacteraemia in Denmark 1980-1990. J Infect 34:113-118, 1997

10. Kang DH, Kim YJ, Kim SH, Sun BJ, Kim DH, Yun SC, et al: Early surgery versus conventional treatment for infective endocarditis. N Engl J Med 366:2466-2473, 2012

11. Kehrer M, Pedersen C, Jensen TG, Lassen AT: Increasing incidence of pyogenic spondylodiscitis: a 14-year populationbased study. J Infect 68:313-320, 2014

12. Le Moal G, Roblot F, Paccalin M, Sosner P, Burucoa C, Roblot $\mathrm{P}$, et al: Clinical and laboratory characteristics of infective endocarditis when associated with spondylodiscitis. Eur J Clin Microbiol Infect Dis 21:671-675, 2002

13. Leroy O, Georges H, Devos P, Bitton S, De Sa N, Dedrie C, et al: Infective endocarditis requiring ICU admission: epidemiology and prognosis. Ann Intensive Care 5:45, 2015
14. Morelli S, Carmenini E, Caporossi AP, Aguglia G, Bernardo ML, Gurgo AM: Spondylodiscitis and infective endocarditis: case studies and review of the literature. Spine (Phila Pa 1976) 26:499-500, 2001

15. Nicholls A, Edward N, Catto GR: Staphylococcal septicaemia, endocarditis, and osteomyelitis in dialysis and renal transplant patients. Postgrad Med J 56:642-648, 1980

16. Ninet J, Gayet JL, Etienne J, Bonvoisin B, Vignon E, Berthou $\mathrm{JD}$, et al: Bacterial endocarditis presenting as acute vertebral osteomyelitis: 14 cases. Eur Heart J 5 (Suppl C):101-105, 1984

17. Petti CA, Fowler VG Jr: Staphylococcus aureus bacteremia and endocarditis. Cardiol Clin 21:219-233, vii, 2003

18. Pigrau C, Almirante B, Flores X, Falco V, Rodríguez D, Gasser I, et al: Spontaneous pyogenic vertebral osteomyelitis and endocarditis: incidence, risk factors, and outcome. Am J Med 118:1287.e17-1287.e24, 2005

19. Sampath P, Rigamonti D: Spinal epidural abscess: a review of epidemiology, diagnosis, and treatment. J Spinal Disord 12:89-93, 1999

20. Sapico FL, Liquete JA, Sarma RJ: Bone and joint infections in patients with infective endocarditis: review of a 4-year experience. Clin Infect Dis 22:783-787, 1996

21. Sullenberger AL, Avedissian LS, Kent SM: Importance of transesophageal echocardiography in the evaluation of Staphylococcus aureus bacteremia. J Heart Valve Dis 14:23-28, 2005

\section{Disclosures}

The authors report no conflict of interest concerning the materials or methods used in this study or the findings specified in this paper.

\section{Author Contributions}

Conception and design: Behmanesh, Gessler, Konczalla. Acquisition of data: Behmanesh, Gessler, Schnoes, Dubinski, Won. Analysis and interpretation of data: Behmanesh, Schnoes. Drafting the article: Behmanesh. Reviewed submitted version of manuscript: Seifert, Weise, Setzer. Approved the final version of the manuscript on behalf of all authors: Behmanesh. Statistical analysis: Behmanesh, Konczalla. Study supervision: Seifert.

\section{Supplemental Information \\ Videos}

Video Abstract. https://vimeo.com/305050340.

\section{Previous Presentations}

Parts of this manuscript was presented at the 69th Annual Meeting of the German Society of Neurosurgery, June 3-6, 2018, in Münster.

\section{Correspondence}

Bedjan Behmanesh: University Hospital, Frankfurt am Main, Germany. bedjan.behmanesh@gmail.com. 